\title{
High throughput in Uplink SC-FDMA Systems with Multicell Processing
}

\author{
Sapna Kumari \\ M.Tech research Scholor \\ Bhopal Institute of \\ Technology Bhopal, \\ MP, India
}

\author{
Bramha S.Tripathi \\ Assistant Professor \\ Bhopal Institute of \\ Technology Bhopal, MP, India
}

\author{
Shilpi Sharma, PhD \\ Associate Professor \\ Bhopal Institute of \\ Technology Bhopal, \\ $\mathrm{MP}$, India
}

\begin{abstract}
In current cellular networks base stations (BSs) usually perform independent scheduling without coordinating the resource allocation among different cells. This, however, often leads to high interference levels in cellular networks operating with universal frequency reuse, such as the 3GPP UTRAN Long Term Evolution (LTE). Coordinated scheduling between different BSs may mitigate this problem by taking interference from and to nearby BSs into account in order to avoid high interference situations. For uplink in multiuser major issue to maintain throughput of the system. To solve this problem, a channel estimation method for SCOFDM under the framework of compressive sensing (CS) is proposed in this paper. Firstly, by exploiting the signal.(SP) algorithm to utilize a very small amount of frequency domain pilots embedded in the SC-OFDM block for the exact channel estimation. Moreover, the obtained auxiliary channel information is adopted to reduce the complexity of the classical SP algorithm..
\end{abstract}

\section{Keywords}

High Throughput, Multiuser,LTE,SC-FDMA,3GPP,Cellular System.

\section{INTRODUCTION}

SC-OFDM is a signaling technique that has been applied widely in wireless communication systems due to its ability to maintain effective transmission and highly efficient bandwidth utilization in the presence of various channel impairments which one of them is frequency selective fading. Recently, interference coordination has emerged as one of the most attractive techniques for combating interference in next generation cellular networks [1]-[5], such as the 3GPP UTRAN Long Term Evolution (LTE), which operates with universal frequency reuse in order to exploit the whole frequency bandwidth in each cell. In SC-OFDM systems the available spectrum are divided into many orthogonal subchannels, which are instantaneously used to data transmission. Also, in this technique the inter-symbol interference (ICI) which is induced due to frequency selective channels can be reduced by adding the cyclic prefix (CP) [1]. In SC-OFDM systems, channel estimation is necessary to obtain the channel state information (CSI), reducing the bit error rate and also to achieve a distortion less output data. There are various methods to channel estimation such as: with or without a need to parametric models, blind or pilot based methods, frequency and/or time domain analysis, adaptive or non adaptive techniques. Among these mentioned methods, channel estimation in SC-OFDM systems is often done in frequency domain using pilot symbols or training data [2]. The least square and minimum mean-square error (MMSE) are conventional linear channel estimation techniques which are based on pilot arrangement. The LS method is less complicated and simple respect to other methods and consequently is used to channel estimation, but it has a serious drawback which is more sensitive to channel noise. MMSE estimator has better performance than LS method but suffers from a high computational complexity because it requires knowledge of the channel statistics and the signal-to-noise ratio (SNR) [3]. Some different methods have been developed to reduce the complexity and improve the performance of the MMSE estimation such as modified MMSE and singular value decomposition (SVD) [4-5]. 1n 2006 Noh et al. proposed a method to decomposing the covariance matrix to the simple and low order sub matrix so that they can decrease the complexity of MMSE method [6]. Hsieh used a comb type pilot arrangement and second order interpolation method to channel estimation [7]. Coleri et al. compared the results of many interpolation techniques to channel estimation with Rayleigh fading such as linear, second order, cubic, low pass filtering and spline interpolation methods The recently introduced principle and methodology of compressed sensing (CS) allows the efficient reconstruction of sparse signals from a very limited number of measurements (samples) [1, 2]. CS has gained a fast-growing interest in applied mathematics. In this paper, we apply CS to pilot-based channel estimation in highly mo-bile environments. We consider pulse-shaping multicarrier (MC) systems, which include orthogonal frequency-division multiplexing (OFDM) as a special case [3]. Conventional methods for channel estimation (e.g., [4]) are not able to exploit the inherent sparsity of the transmission channel that is due to the sparse distribution of scat-terers in space. As we will demonstrate, CS provides a constructive way for exploiting this sparsity in order to reduce the number of pi-lots and, hence, increase spectral efficiency.

Radio transmission has allowed people to communicate without any physical connection for more than hundred years. When Marconi managed to demonstrate a technique for wireless telegraphy, more than a century ago, it was a major breakthrough and the start of a completely new industry. May be one could not call it a mobile wireless system, but there was no wire! Today, the progress in the semiconductor technology has made it possible, not to forgot affordable, for millions of people to communicate on the move all around the world.

The Mobile Communication Systems are often categorized as different generations depending on the services offered. The first generation comprises the analog frequency division multiple access (FDMA) systems such as the NMT and AMPS (Advanced Mobile Phone Services). The second generation consists of the first digital mobile communication systems such as the time division multiple access (TDMA) based GSM (Global System for Mobile 
Communication), D-AMPS (Digital AMPS), PDC and code division multiple access (CDMA) based systems such as IS95. These systems mainly offer speech communication, but also data communication limited to rather low transmission rates. The third generation started operations on 1st October 2002 in Japan.

During the past few years, there has been an explosion in wireless technology. This growth has opened a new dimension to future wireless communications whose ultimate goal is to provide universal personal and multimedia communication without regard to mobility or location with high data rates. To achieve such an objective, the next generation personal communication networks will need to be support a wide range of services which will include high quality voice, data, facsimile, still pictures and streaming video. These future services are likely to include applications which require high transmission rates of several Mega bits per seconds (Mbps).

In the current and future mobile communications systems, data transmission at high bit rates is essential for many services such as video, high quality audio and mobile integrated service digital network. When the data is transmitted at high bit rates, over mobile radio channels, the channel impulse response can extend over many symbol periods, which leads to inter symbol interference (ISI). Orthogonal Frequency Division Multiplexing (OFDM) is one of the promising candidates to mitigate the ISI. In an OFDM signal the bandwidth is divided into many narrow sub channels which are transmitted in parallel. Each sub channel is typically chosen narrow enough to eliminate the effect of delay spread. By combining OFDM with Turbo Coding and antenna diversity, the link budget and dispersive-fading limitations of the cellular mobile radio environment can be overcome and the effects of co-channel interference can be reduced.

\section{MODULE}

\subsection{LTE}

Long-Term Evolution (LTE) is the air interface supporting fourth generation cellular networks. LTE is specifically designed for packet data communications, where the emphasis of the technology is high spectral efficiency, high peak data rates, low latency, and frequency flexibility. The LTE specifications were developed by the Third Generation Partnership Project (3GPP). GSM and UMTS are the predecessors of the LTE air interface and are referred to as second generation (2G) and third generation (3G) technologies, respectively. GSM was developed as a circuit switched network meaning that radio services are configured at the user's request and resources remain allocated until terminated by the network controller. This type of operation is well suited to supporting voice calls. Eventually, GSM was enhanced to support low data rate services with packet switching capability but data rates were limited by GSM's air interface, time division multiple access (TDMA). In TDMA, each user is assigned to a particular channel (frequency band) and time slot which serves to limit capacity as the channel spacing is only $200 \mathrm{kHz}$.

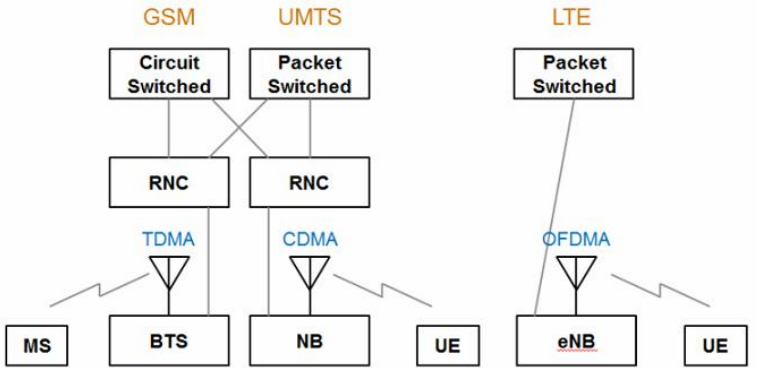

Fig 1 LTE cellular network

\subsection{SC-FDMA}

The complex data symbols are modulated by means of an inverse discrete Fourier transform (IDFT) on $\mathrm{N}$ parallel subcarriers. The resulting SC-OFDM symbol is serially transmitted over a discrete-time channel, whose impulse response we assume is shorter than $\mathrm{L}$ samples. At the receiver, the data are retrieved by means of a discrete Fourier transform(DFT) An accepted means of avoiding inter symbol interference (ISI) and preserving orthogonality between subcarriers is to copy the last $\mathrm{L}$ samples of the body of the $\mathrm{SC}-\mathrm{OFDM}$ symbol ( $\mathrm{N}$ samples long) and append them as a preamble | the cyclic prefix | to form the complete SC-OFDM symbol. The effective length of the SC-OFDM symbol as transmitted is this cyclic prefix plus the body $(\mathrm{L}+\mathrm{N}$ samples long). The insertion of a cyclic prefix can be shown to result in an equivalent parallel orthogonal channel structure that allows for simple channel estimation and equalization. In spite of the loss of transmission power and bandwidth associated with the cyclic prefix, these properties generally motivate its use.

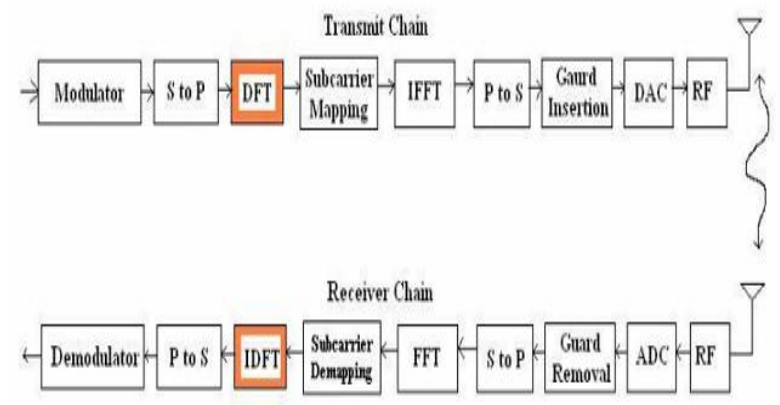

Fig: 2 SC-OFDM systems modal

Without a frequency offset, the frequency response of each sub channel is zero at all other subcarrier frequencies, i.e., the sub channels do not interfere with one other. The effect of a frequency offset is a loss of orthogonality between the tones. The resulting inter carrier interference (ICI) has been investigated. The effective signal-to-noise ratio (SNR) due to both additive noise and ICI is shown to be lower bounded by

$$
S N R_{e}(\varepsilon) \geq \frac{S N R}{1+0.54947 S N R \sin ^{2} \pi \varepsilon}\left(\frac{\sin \pi \varepsilon}{\pi \varepsilon}\right)^{2}
$$

\subsection{Channel Estimation Based On Block- Type Pilot Arrangement}

In block-type pilot based channel estimation, OFDM channel estimation symbols are transmitted periodically, in which all sub-carriers are used as pilots. If the channel is constant during the block, there will be no channel estimation error since the pilots are sent at all carriers. The estimation can be performed by using either LSE or MMSE [23], [34]. If inter 
symbol interference is eliminated by the guard interval, we write (3.7) in matrix notation:

$$
\begin{aligned}
Y & =X F h+W \\
& =X H+W
\end{aligned}
$$

\section{METHOD}

Compressive Sensing Orthogonal Frequency Division Multiplexing (CS-OFDM), developed by Cisco, is an open standard for broadband wireless Internet services. CS-OFDM increases subscriber coverage for high-speed, high-reliability Internet, packet local and long distance telephony services and Virtual Private Network (VPN) access. CSOFDM also lowers the cost of provisioning and deploying infrastructure for a wireless network, and utilizes multipath signals to enhance or recreate transmitted signals, dramatically increasing overall system performance and scalability for service providers. This allows for high data throughput in a minimum of Radio Frequency (RF) spectrum and supports extreme multipath issues existing in obstructed or partially obstructed non-lineof-sight environments. Similar to conventional OFDM, in CSOFDM, the modulated symbols are processed block-by-block. Assume that there are $\mathrm{N}=\mathrm{LM}$ modulated symbols in one block. Different from conventional OFDM, CS-OFDM further divides the length $\mathrm{N}$ block into Vector Block (VB), where each VB has size $M$. Instead of doing IFFT of size $N$ as in conventional OFDM, CS-OFDM does component wise vector IFFT of size over the VBs. The IFFT size is reduced from $\mathrm{N}$ to $\mathrm{L}$ by $\mathrm{M}$ times. This IFFT size reduction also reduces the PAPR.it has the merit of Low PAPR and Cost reduction for transceiver architecture.

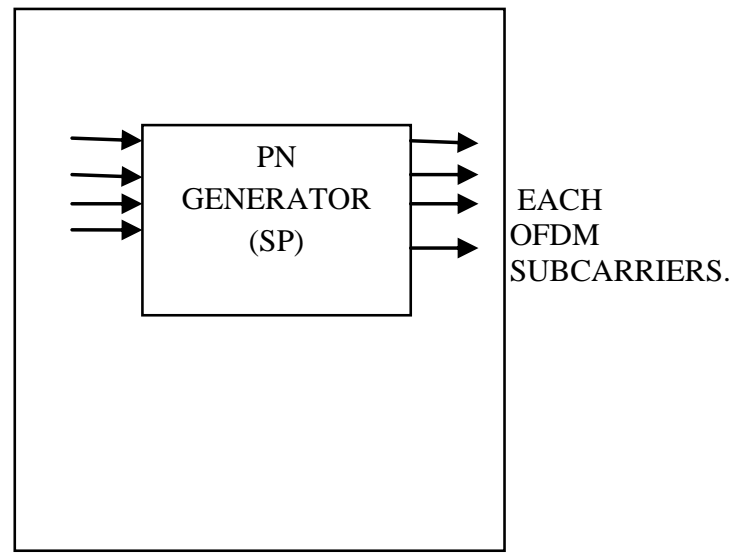

Using TFT we construct the channel parameters and estimate for transmission signal to receiver.

\section{RESULT}

The Complexity Order of A-SP for SARFT-8 channel $1.539269 \mathrm{e}+01$. The Complexity Order of A-SP for IYU-VB channel 1.584223e+01. The Complexity Order of SP $1.471766 \mathrm{e}+01$.
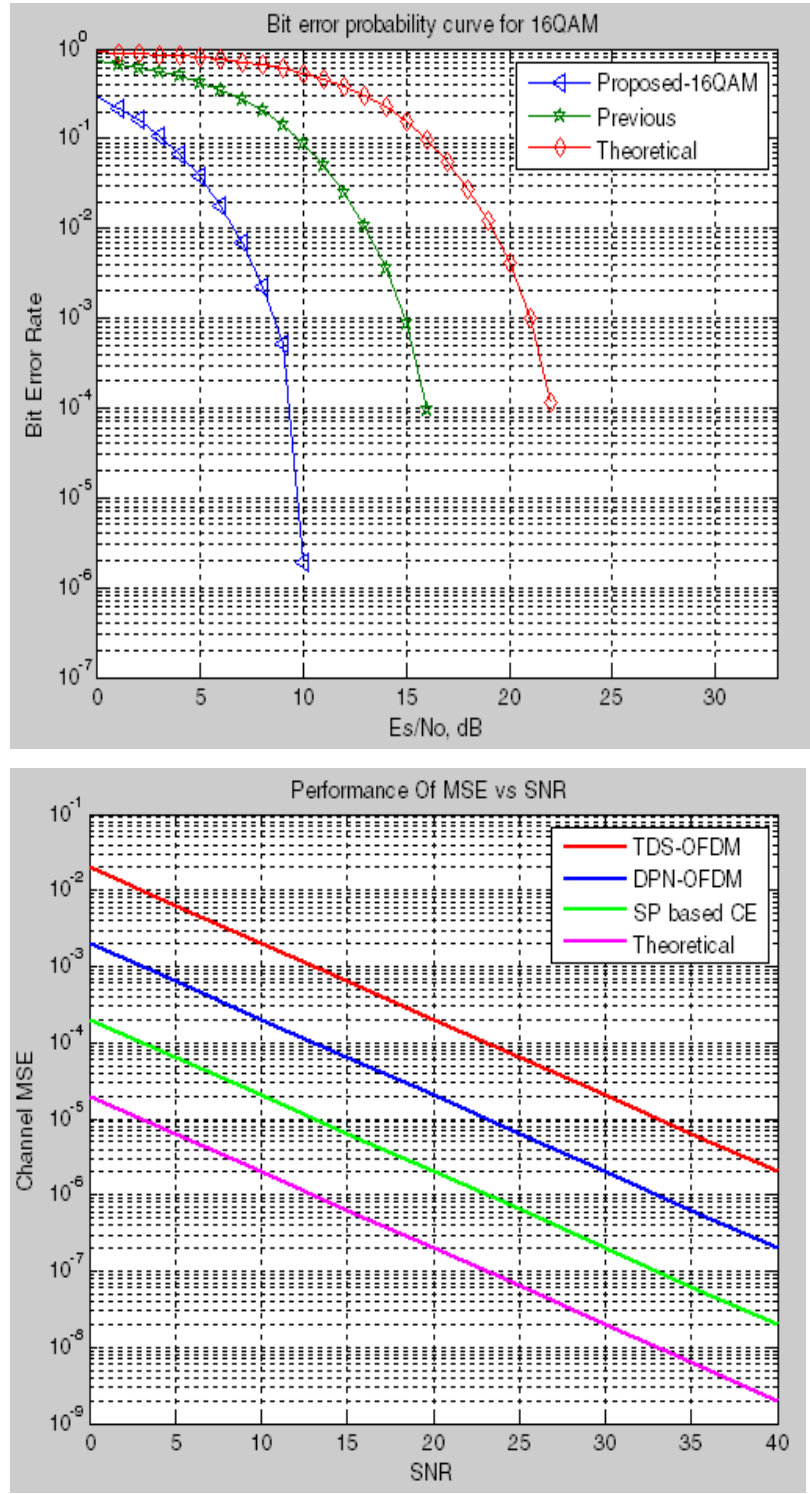

Fig:3 Performance of MSE vs SNR

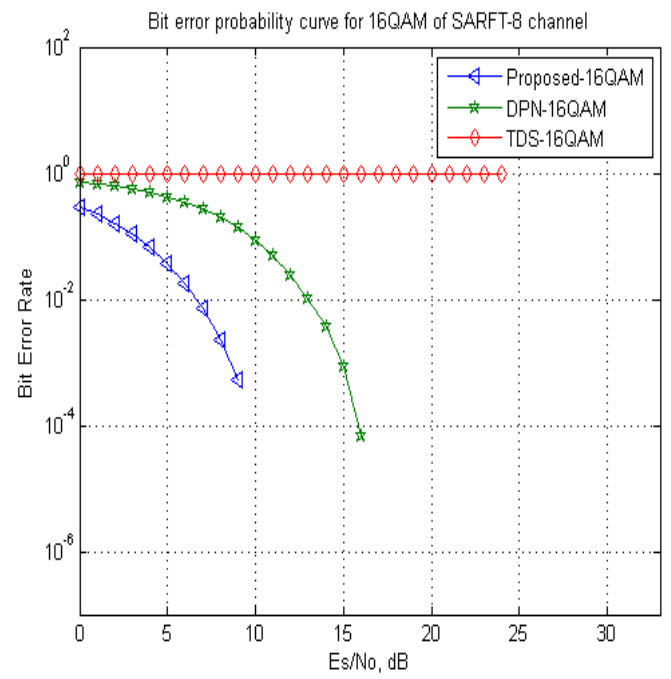

Fig: 4 BEC for 16QAM 


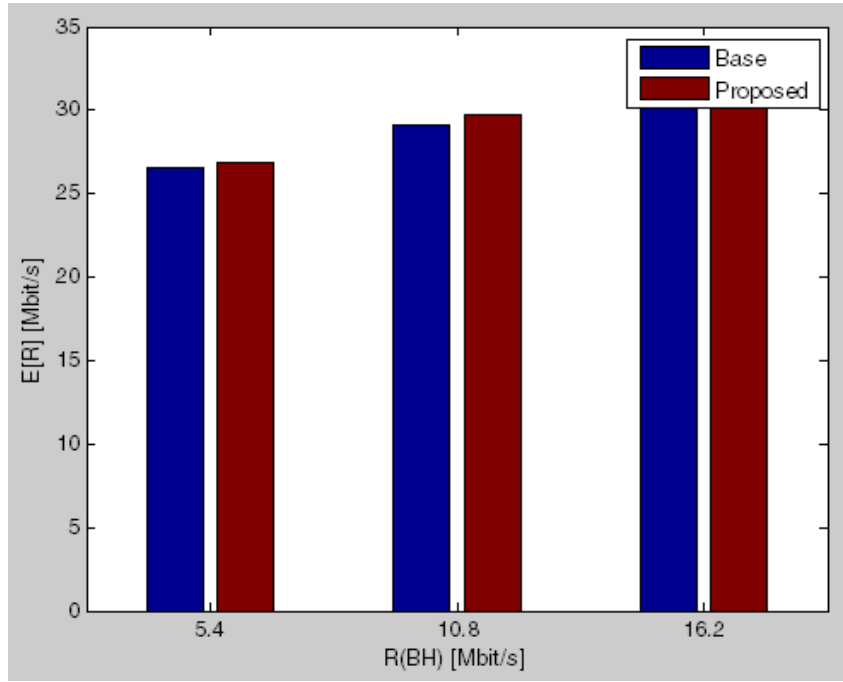

Fig : 5 Throughput

\section{CONCLUSION}

In this paper proposed we have proposed a CS-based CE method for high data throughput. We have developed a resource allocation algorithm for the scheduling of backhaul transmissions. By considering an efficient composite bit representation of different quantized versions of received signals, the proposed algorithm iteratively schedules the subcarrier signal providing the maximum network throughput increase per backhaul bit.

\section{REFERENCES}

[1] Paolo Baracca, Stefano Tomasin, M, and Nevio Benvenuto, "Backhaul Rate Allocation in Uplink SCFDMA Systems with Multicell Processing" IEEE Tran. on Wireless Comm., vol. 13, no. 3, pp. 1264-1273 March 2014.

[2] C. Han, Tim Harrold, and S. Armour,. "Green Radio: Radio Techniques to Enable Energy-Efficient Wireless Networks" IEEE Commu. Magazine pp. 46-54, 2011

[3] J. Zhang, L.Yang, and L. Hanzo," "Energy-Efficient Channel-Dependent Cooperative Relaying for the MultiUser SC-FDMA Uplink," IEEE Tran. on Vol..60 , Iss.. 3 pp. 992 - 1004, March 2011.

[4] P. Frank, A. Muller, H. Droste, and Joachim Speidel, "Cooperative Interference-Aware Joint Scheduling for the 3GPP LTE Uplink" IEEE 21st Inte. Symp. Pp. 2216 -2221, 26-30 Sept. 2010

[5] J. N. Laneman, D. N. C. Tse, and G. W. Wornell, "Cooperative diversity in wireless networks: Efficient protocols and outage behavior," IEEE Transactions on Information Theory, vol. 50, no. 12, pp. 3062-3080, Dec. 2004.

[6] W. Fang, L.-L. Yang, and L. Hanzo, "Single-user performance of direct sequence code-division multipleaccess using relay diversity and power allocation," IET Proceedings on Communications, vol. 2, no. 3, pp. 462472, Mar. 2008.

[7] R. Zhang and L. Hanzo, "Coding schemes for energy efficient multisource cooperation aided uplink transmission," IEEE Signal Processing Letters, vol. 16, no. 5, pp. 438-441, May 2009.
[8] L. Wang and L. Hanzo, "The resource-optimized differentially modulated hybrid AF/DF cooperative cellular uplink using multiple-symbol differential sphere detection," IEEE Signal Processing Letters, vol. 16, no. 11, pp. 965-968, Nov. 2009.

[9] Y. Li, B. Vucetic, Z. Zhou, and M. Dohler, "Distributed adaptive power allocation for wireless relay networks," IEEE Transactions on Wireless Communications, vol. 6, no. 3, pp. 948-958, Mar. 2007.

[10] [M. Kaneko, K. Hayashi, P. Popovski, K. Ikeda, H. Sakai, and R. Prasad, "Amplify-and-forward cooperative diversity schemes for multi-carrier systems," IEEE Transactions on Wireless Communications, vol. 7, no. 5, pp. 1845-1850, May 2008.

[11] E. C. van der Meulen, "Three-terminal communication channels," Advances in Applied Probability, vol. 3, no. 1, pp. 120-154, 1971.

[12] A. Bletsas, A. Khisti, D. Reed, and A. Lippman, "A simple cooperative diversity method based on network path selection," IEEE Journal on Selected Areas in Communications, vol. 24, no. 3, pp. 659-672, Mar. 2006.

[13] Y. Zhao, R. Adve, and T. J. Lim, "Improving amplifyand-forward relay networks: Optimal power allocation versus selection," IEEE Transactions on Wireless Communications, vol. 6, no. 8, pp. 3114-3123, Aug. 2007.

[14] A. Bletsas, H. Shin, and M. Z. Win, "Cooperative communications with outage-optimal opportunistic relaying," IEEE Transactions on Wireless Communications, vol. 6, no. 9, pp. 3450-3460, Sep. 2007.

[15] R. Madan, N. Mehta, A. Molisch, and J. Zhang, "Energyefficient cooperative relaying over fading channels with simple relay selection," IEEE Transactions on Wireless Communications, vol. 7, no. 8, pp. 3013-3025, Aug. 2008.

[16] Y. Jing and H. Jafarkhani, "Single and multiple relay selection schemes and their achievable diversity orders," IEEE Transactions on Wireless Communications, vol. 8, no. 3, pp. 1414-1423, Mar. 2009.

[17] H. G. Myung, J. Lim, and D. J. Goodman, "Single carrier FDMA for uplink wireless transmission," IEEE Vehicular Technology Magazine, pp.30-38, Sep. 2006.

[18] E-UTRA LTE Physical layer - General description, 3GPP Std. TS 35.201(V8.3.0), 2009.

[19] D. Falconer, S. L. Ariyavisitakul, A. Benyamin-Seeyar, and B. Eidson, "Frequency domain equalization for single-carrier broadband wireless systems," IEEE Communications Magazine, pp. 58-66, Apr. 2002.

[20] N. Benvenuto, R. Dinis, D. Falconer, and S. Tomasin, "Single carrier modulation with nonlinear frequency domain equalization: An idea whose time has comeagain," Proceedings of the IEEE, vol. 98, no. 1, pp. 69 96, Jan. 2010

[21] F. Pancaldi, G. M. Vitetta, R. Kalbasi, N. Al-Dhahir, M. Uysal, and H. Mheidat, "Single-carrier frequency domain equalization," IEEE Signal Processing Magazine, pp. 37-56, Sep. 2008. 
[22] L. Hanzo, M. M“unster, B.-J. Choi, and T. Keller, OFDM and MC-CDMA for Broadband Multi-User Communications, WLANs and Broadcasting. Wiley, 2003.

[23] H. G. Myung and D. J. Goodman, Single Carrier FDMA: A New Air Interface for Long Term Evolution. Wiley, 2008 .

\section{AUTHOR PROFILE}

Sapna Kumari is a M-Tech.(Digital Communication) student and a research scholar in the Department of Electronics \& Communication from Bhopal Institute of Technology , Bhopal, affiliated to RGPV Bhopal, Madhya Pradesh, India. She received the B.E. degree from RGPV University MP in year 2013, her current research interests are in communication protocols, OFDM.

Prof. Bramha S. Tripathi, is an Assistant Professor at the Department of Electronics \& Communication, Bhopal Institute of Technology, Bhopal. His research interest is in the area of Wireless communication, optical communication and VLSI Design. I have completed my M-Tech .in VLSI Design from L.N.C.T.college affiliated to RGPV Bhopal (M.P.), India in year 2013 with Honors degree. I have awarded an Honors B.E. degree from RGPV, Bhopal (M.P.) in year 2009. I have completed Diploma engineering in Electronics \& Telecommunication from RGPV, Bhopal (M.P.) in 2006. 